\title{
Miranda
}

Revue pluridisciplinaire du monde anglophone /

Multidisciplinary peer-reviewed journal on the English-

speaking world

$12 \mid 2016$

Mapping gender. Old images ; new figures

\section{De la musique, de la mer, des mots pour les dire : interview de Christophe Hardy, écrivain, poète et musicien}

\author{
Nathalie Vincent-Arnaud
}

\section{OpenEdition}

Journals

Édition électronique

URL : http://journals.openedition.org/miranda/8511

DOI : 10.4000/miranda. 8511

ISSN : 2108-6559

\section{Éditeur}

Université Toulouse - Jean Jaurès

\section{Référence électronique}

Nathalie Vincent-Arnaud, « De la musique, de la mer, des mots pour les dire : interview de Christophe Hardy, écrivain, poète et musicien », Miranda [En ligne], 12 | 2016, mis en ligne le 01 mars 2016, consulté le 16 février 2021. URL : http://journals.openedition.org/miranda/8511 ; DOI : https://doi.org/ $10.4000 /$ miranda. 8511

Ce document a été généré automatiquement le 16 février 2021.

\section{c)}

Miranda is licensed under a Creative Commons Attribution-NonCommercial-NoDerivatives 4.0

International License. 


\title{
De la musique, de la mer, des mots pour les dire : interview de Christophe Hardy, écrivain, poète et musicien
}

\author{
Nathalie Vincent-Arnaud
}

1 Thomas Adès, George Benjamin, Luciano Berio, Hector Berlioz, Frank Bridge, Gilles Bonnet, Benjamin Britten, William Byrd, John Cage, Sir Colin Davis, Alfred Deller, John Dowland, Edward Elgar, Georg Friedrich Haendel, Felicity Lott, Robert Pinget, Francis Poulenc, Henry Purcell, Arthur Rimbaud, Jean-François Sivadier, Michael Tippett, Jon Vickers, Richard Wagner, Vaughan Williams

2 Écrivain, poète, musicien (pianiste et chanteur lyrique) ayant longuement pratiqué la danse, ancien élève de l'ENS, contributeur régulier de la revue de culture générale L'Éléphant ( http://www.lelephant-larevue.fr/christophe-hardy/), Christophe Hardy conjugue avec bonheur des passions et des talents diversifiés qui donnent régulièrement naissance à des ouvrages où humour et plaisir de l'écriture le disputent à l'érudition soignée du propos. Son opus Les Mots de la musique (Paris, Belin, 2007) est une parfaite illustration de son écriture abondamment nourrie d'un enthousiasme encyclopédique qu'il a à cœur de cultiver et de faire partager. Et la sphère anglosaxonne y figure en bonne place.

Nathalie Vincent-Arnaud: Tu as signé à ce jour de nombreux ouvrages qui vont de la poésie aux études sur des écrivains et des musiciens, en passant par Les Mots de la musique (paru chez Belin en 2003) mais aussi par des livres qui ont pour but d'initier à l'art (aux éditions Le Baron perché). On pourrait parler d'éclectisme mais je qualifierais plutôt, pour avoir lu un certain nombre de tes écrits, ta démarche d'englobante. Cet adjectif te semble-t-il pertinent, et pourrais-tu préciser la manière dont tu conçois toi-même cette diversité ?

Christophe Hardy: Ces livres se fondent sur une culture personnelle, qui a ses richesses et ses limites. Ils reflètent ma familiarité avec un certain nombre de domaines : la littérature, la poésie, la langue, la musique, la danse, le théâtre, les beaux-arts; et mon désir d'en approfondir la connaissance. Ils sont ce que j'appelle 
des «livres de savoir» pour les distinguer de quelques autres qui, eux, sont des « livres de création ». Je fais en sorte de les régler toujours sur le même principe : armer le lecteur pour qu'il puisse, avec son envie, sa persévérance, s'aventurer à l'intérieur d'une œuvre, en approcher les mystères. S'il y a quelque chose d'englobant dans ma démarche, elle n'a - j'espère - rien de totalitaire : je ne cherche pas avoir le mot de la fin. J'abandonne toujours le lecteur beaucoup plus tôt. J'ai souvent l'image de quelqu'un que je guide fraternellement à l'orée d'une forêt, où moi-même me suis déjà risqué, mais je ne le conduis pas au-delà. À lui de s'enfoncer dans les épaisseurs qu'il entrevoit.

NVA: Si je fais retour aux Mots de la musique, quelle a été ta principale motivation dans l'écriture de ce livre? On est frappé, à sa lecture, par le mélange d'érudition du propos, de jubilation dans le ton et d'ouverture vers un large public.

$\mathrm{CH}$ : La première et principale motivation : faire un livre qui n'existait pas! Je savais que je me lançais dans un chantier de longue haleine, j'avais l'expérience d'un précédent dictionnaire thématique, Les Mots de la mer, publié chez le même éditeur, en 2002. L'essentiel était de ne pas s'essouffler, de maintenir le plaisir de la découverte. Ce plaisir que j'ai pris pendant le temps du travail de construction (collectage, tri des informations) et d'écriture, j'espère qu'on le ressent à la lecture. Même si j'ai procédé de façon systématique, lettre après lettre - pas forcément dans l'ordre de l'alphabet car il y a des lettres «redoutables» en français, le C par exemple, très décourageant tant il y a d'entrées à traiter... - je brisais la routine en introduisant une variété d'approches, en jouant avec les citations, avec la longueur des entrées (quelques lignes ou trois pages), en choisissant un thème insolite, un traitement "décalé » (je pense à des entrées comme "applaudir - ou ne pas applaudir ", " chat ", " punto Callas », «toux», "yaourt», " wagnérien») ou en m'intéressant à des noms propres (lieux, personnages mythologiques, institutions musicales et autres).

NVA : Sans grande surprise au vu de ta formation et de tes domaines d'intérêt, tu accordes une place importante aux autres arts dans cet ouvrage. Quelle est selon toi la place de la musique dans le panorama des arts et dans la réflexion que l'on peut conduire, d'une manière générale, sur l'art?

$\mathrm{CH}$ : Ce livre reflète différents aspects de ma formation (études de lettres, études musicales en parallèle, pratique de la danse, du chant et de plusieurs instruments) et, dans le prolongement de cette formation, mes sujets de prédilection : je puise dans ce qui m'est familier, la poésie, le monde de la danse et de l'art lyrique, la musique vocale sous de multiples formes, de la comptine au bel canto en passant par la chanson traditionnelle et par les expériences sur la voix d'un Berio ou d'un Cage.

Deux points pour répondre (plus ou moins bien) à ta question.

1/ Plus que la place de la musique dans le panorama des arts, m'intéresse la question d'installer au centre de l'éducation la pratique de la musique et celle d'autres arts. Je les considère - la pratique de la musique en particulier - comme disciplines nécessaires/bienfaisantes pour la formation/l'entretien du corps et de l'esprit. Dit comme ça, c'est schématique - ça appellerait des développements plus fins, plus précis. Aborder la musique à travers le jeu instrumental, la voix, la simple écoute ou encore à travers la danse (le geste chorégraphique, autre façon de vivre la musique), cela enrichit les rapports que l'on entretient avec le temps, avec l'espace, avec l'autre et avec soi-même. Cela couvre un vaste champ d'expressions, qui vont de formes très 
spontanées à des formes plus élaborées pour lesquelles sont requis l'apprentissage long, la concentration, la patience.

2/ Je pars du principe que les mots permettent de mieux approcher les arts, d'en approfondir la jouissance - je suis assez peu adepte, pour ce qui est de l'émotion esthétique, des catégories «ineffable» et «indicible». Dans l'expérience que constitue la rencontre d'une œuvre d'art, il y a ce moment inaugural, que je trouve magnifique (fraîcheur du saut dans l'inconnu ou de l'attente de quelque chose d'inouii) : moment du silence intérieur (se taire devant une tableau, se concentrer sur l'écoute d'une symphonie ou d'un quatuor à cordes...). C'est là que l'on accorde à une œuvre d'art le temps de faire son chemin jusqu'à nous, d'imposer sa singularité et son étrangeté. Vient ensuite le moment de la prise de parole : mettre des mots sur des sensations, poser des mots sur une œuvre d'art non pas comme si on jetait un voile sur elle - le langage a souvent, malheureusement, cette fonction d'obscurcir l'œuvre, de l'effacer, d'empêcher qu'elle ne surgisse comme un événement singulier, perturbant, chargé de mystère - mais pour prendre acte du fait, à travers le mot/ la formulation justes, que la rencontre a eu lieu.

NVA : À l'inverse d'une tendance affirmée dans certains dictionnaires ou encyclopédies, ton ouvrage t'amène à te colleter avec tous les genres musicaux, de la musique « savante » à la musique «populaire» (pour utiliser des étiquettes consacrées), et on note ainsi des développements sur le groove, le protest song, le hip hop. Ces entrées témoignent-elles d'un souci avant tout civilisationnel ou proprement musical, ou bien les deux dimensions s'articulent-elles pour toi tout naturellement?

$\mathrm{CH}$ : Plusieurs principes à la source du livre. 1/ Je n'ai pas trop le culte de l'expertise et j'ai beaucoup de nostalgie pour l'esprit encyclopédique... Ce qui veut dire que pour certains genres musicaux, certaines notions faisant partie du paysage, il a fallu que je me documente, que je fasse de sérieuses séances de rattrapage. Il était hors de question que j'exerce une forme de censure au nom de mes goûts. 2/ Je rêve d'un lecteur qui, quel que soit son rapport à la musique, ait en lisant l'ouvrage (évidemment ni dans l'ordre ni dans sa totalité) l'envie d'aller vers des domaines qui lui étaient inconnus. Ce livre, comme tous les autres que je conçois, est ouvert sur d'autres livres (l'importance des sources! de la bibliographie! Dire toujours d'où l'on vient...). Les Mots de la musique est ouvert sur une pluralité d'objets esthétiques : des livres bien sûr (ouvrages de littérature, romans, poèmes, etc. - via les citations) mais aussi des œuvres musicales ou des pratiques évoquées au gré des entrées.

NVA: Les références littéraires abondent particulièrement dans ce livre, du roman au théâtre en passant par la poésie et par d'autres formes dérivées. Les rapports entre la musique et la littérature sont multiples comme on le sait, mais quelles orientations particulières as-tu privilégié dans ces rapports, quel traitement leur réserves-tu dans ce livre?

$\mathrm{CH}$ : J'ai suivi plusieurs pistes. D'abord, je tenais à ce qu'il y ait beaucoup de citations, et de toutes sortes. D'où un travail de collectage important. Ensuite s'est posée la question de la place des citations dans les entrées. Classiquement une brève citation vient illustrer un sens qui vient d'être donné. J'ai procédé différemment. J’ai pratiqué une poétique de la citation assez instinctive, en variant la longueur des extraits cités (ils sont parfois longs, d'autres fois lapidaires), en n'illustrant pas systématiquement tous les sens d'un mot, en plaçant des citations en ouverture d'entrée (surtout si elles sont belles ou frappantes). Lors du collectage, j'ai tenu à ménager une place à des types de texte très divers - journaux, chansons, correspondances... - au nom du 
principe que les mots de la musique appartiennent à la langue de tous les jours. Mais j'ai pris soin d'accorder une place de choix à la prose littéraire et à la poésie - la langue des "jours de fête"! Enfin, je me suis volontiers appuyé sur les textes d'écrivains musiciens, au premier rang desquels Berlioz. C'est un auteur que j'ai beaucoup fréquenté, pas seulement comme mélomane. Lorsque j'étais étudiant en lettres, à une époque où Berlioz n'avait pas encore retrouvé l'aura qu'il mérite (sauf en Angleterre, grâce à Sir Colin Davis), j'ai travaillé sur ses écrits, les Mémoires, les fictions, la correspondance, et en particulier sur les rapports de l'artiste avec l'Italie, une Italie réelle autant que rêvée. On trouve aussi un certain nombre de citations de Poulenc. Enfin, il y a des citations que j'ai choisies délibérément pour faire connaître une œuvre, un auteur - par exemple la pièce Italienne, scène et orchestre de JeanFrançois Sivadier a été une source où j'ai puisé pas mal de citations désopilantes. Je pourrais dire ça également des Soirées de l'orchestre de Berlioz.

NVA : Ces dernières années, on a beaucoup parlé de chanson «populittéraire » (titre d'un ouvrage collectif paru chez Kimé en 2013, dirigé par Gilles Bonnet). On évoque ainsi, en vrac, la chanson traditionnelle mais aussi des formes nées dans le monde anglo-saxon telles que le slam, le rap, etc. Ce terme est-il pertinent selon toi et quelles résonances peut-il avoir de nos jours?

$\mathrm{CH}$ : Je ne connais pas la chanson "populittéraire ", tu m'expliqueras. Globalement, le travail sur Les Mots de la musique m'a fait m'interroger sur le sens et la pertinence des «étiquettes». Étant donné que la pensée procède par "entrées» (principe du dictionnaire), j'ai dû réfléchir sur le sens de certaines d'entre elles et sur leurs contenus : «baroque " ça commence quand ? ça finit quand? «classique » recouvre quelle période ? est-ce que le contraire de la musique «savante » c'est la musique « ignorante »? est-ce que le complément de la musique "légère » est la musique « lourde »? pourquoi parle-t-on d' " alto » (« haut » en italien) pour la voix de femme la plus grave/ basse ? À force de segmenter les styles musicaux en sous-catégories dans le domaine du jazz, de la pop etc. est-ce que les mots (qui virent au jargon pour initiés) ne finissent pas par se vider d'un contenu réel ? etc. Ces questions, et bien d'autres encore, invitent par l'éclairage porté sur les mots, à remettre en question (ou à authentifier) catégories et définitions. Ça fait comprendre combien le réel (musical) est riche, mouvant, en perpétuelle métamorphose avec, pour le saisir aussi largement que possible, le filet des mots qui, bien sûr, laisse toujours passer du vivant entre les mailles.

NVA: Quelle place occupe la musique anglaise dans ton livre? On a souvent évoqué la Renaissance comme étant son «âge d'or »; es-tu d'accord avec cette conception, ou bien vois-tu un degré d'accomplissement similaire à d'autres époques?

$\mathrm{CH}$ : Dans Les Mots de la musique, la principale «musique anglaise » est celle des mots, des emprunts que le français fait au monde anglo-saxon, en puisant volontiers à cette source-là pour décrire des réalités musicales contemporaines, jazz, rock, pop music, musiques électroniques... qui occupent une place considérable dans la culture et l'économie mondiales depuis le milieu du XX $\mathrm{XX}^{\mathrm{e}}$ siècle. Pour ce qui est de la musique anglaise dite "savante ", j'en parle ici en tant que mélomane et musicien. C'est vrai que les $\mathrm{XVI}^{\mathrm{e}}, \mathrm{XVII}^{\mathrm{e}}$ et $\mathrm{XVIII}{ }^{\mathrm{e}}$ siècles sont une période faste, avec de grandes figures, Byrd, Dowland, Purcell, Haendel. Je ne peux que donner des exemples personnels : j'ai découvert Dowland grâce aux enregistrements d'Alfred Deller, Purcell et Haendel grâce à des enregistrements de John Eliot Gardiner (me revient en mémoire le très beau L'Allegro, il penseroso ed il moderato). Mais le $\mathrm{XX}^{\mathrm{e}}$ siècle est, je trouve, presque 
aussi riche, dominé par la figure de Britten. Je l'ai découvert grâce à la poésie française puisque mon premier contact avec lui s'est produit en écoutant Illuminations, mélodies avec orchestre sur des poèmes de Rimbaud. Ensuite, il y a eu les opéras, Peter Grimes (incarné par Jon Vickers), Billy Bud, A Midsummer Night's Dream... Avant lui, il y a Elgar, Vaughan Williams, Bridge, Tippett, aujourd'hui George Benjamin, Thomas Adès.

NVA : En amoureux de l'opéra et de l'art lyrique en général, tu offres dans cet ouvrage des développements passionnants sur la voix qui mêlent considérations sur la technique et sur l'expressivité. Tu es toi-même fortement investi dans le chant. Existe-t-il pour toi des approches très différentes du chant selon la langue qu'on utilise, et que peux-tu nous dire en particulier sur l'anglais?

$\mathrm{CH}$ : Parmi les langues que le chant classique m'a fait pratiquer régulièrement, il y a l'italien, l'allemand, le français et l'anglais (un peu moins que les trois autres). L'italien et l'allemand sont plutôt bien adaptés à une technique classique. L'anglais, je ne le trouve pas facile mais sans doute parce que je ne suis pas très à l'aise avec cette langue que, par ailleurs, je trouve très belle à écouter. Ce sont là des considérations subjectives. J'ai entendu l'autre jour à la radio la cantatrice britannique Felicity Lott qui disait avoir beaucoup plus de facilité et de plaisir à chanter en français et en allemand que dans sa langue maternelle. Pour ce qui me concerne, chanter en français est difficile. C'est une langue dont la fluidité me plaît beaucoup, mais quand on passe au chant il faut quasiment réinventer une façon de formuler les sons, voyelles et consonnes, pour obtenir l'ampleur et la projection qu'une technique vocale classique exige.

NVA: Ton livre offre également de beaux développements sur le jazz, mêlés également, parfois, de citations littéraires. Écriture jazz, langue «jazzée », ces deux notions reviennent fréquemment depuis plusieurs années sous la plume de deux qui étudient les rapports entre musique et littérature. Que t'évoquent-elles et quel est à ton sens le degré de proximité des deux langages?

$\mathrm{CH}$ : Je ne connais pas suffisamment ce domaine pour te répondre avec précision. Ce que je sais d'expérience, en tant que lecteur, c'est que les tentatives de transposition de formes musicales en littérature ne m'ont, pour l'instant, jamais convaincu - je me souviens par exemple de Passacaille de Robert Pinget, que j'avais trouvé décevant par rapport à d'autres textes du même auteur.

NVA : Les Mots de la musique, Les Mots de la mer, un effet de parallélisme qui n'est peut-être pas fortuit... Les Anglais sont de grands marins, et la mer étant un thème fréquent dans la littérature et la musique anglo-saxonne (de la ballade populaire aux formes plus "savantes"), il est particulièrement intéressant pour une angliciste de te demander s'il existe selon toi des points de rencontre entre les deux, et lesquels.

$\mathrm{CH}:$ Je te répondrai en deux temps. D'abord une réponse « technique » : quand on fait des dictionnaires, c'est amusant de relever les emprunts aux langues voisines, et d'y réfléchir. Pour Les Mots de la mer, l'arrivage des mots anglais dans le vocabulaire français reflète une période faste de l'histoire maritime britannique (le XIX ${ }^{\mathrm{e}}$, l'apogée de l'Empire et la domination sur les mers) et l'invention de nouvelles pratiques (le yachting). Pour Les Mots de la musique, j'ai déjà évoqué le point: l'arrivage des mots anglo-saxons au $\mathrm{XX}^{\mathrm{e}}$ correspond au succès de formes musicales qui connaissent une diffusion planétaire (jazz, rock, pop, rap...)

Ensuite, mer et musique sont deux « espaces » que j'affectionne. Ils sont propices à l'expansion des sens, de l'esprit, de la rêverie. Ce sont des espaces inspirants et 
exaltants par leur grandeur et par leur pouvoir de dépaysement. Ils exercent une force d'attraction considérable en nous conduisant là où on n'imaginait pas pouvoir aller. Parfois, ça devient troublant quand, au plaisir sonore, s'ajoute la sensation que le discours musical nous piège - je pense, en particulier, à l'écoute live de certains opéras de Wagner - et qu'il nous impose sa présence envahissante. De quoi jouit-on alors? D'un sentiment de plénitude ou d'un sentiment d'écrasement? De la sensation d'être hors de soi, comme arraché à sa condition?

INDEX

Keywords : fine arts, singing, ballet, language, music, sea, poetry, voice

Mots-clés : beaux-arts, chant, danse, langue, musique, mer, poésie, voix

\section{AUTEUR}

\section{NATHALIE VINCENT-ARNAUD}

Professeur

Université Toulouse-Jean Jaurès

nathalie.vincent-arnaud@univ-tlse2.fr 